\title{
ON A REDUCED ORDER FE-MODEL TO SIMULATE NONLINEAR MATERIAL RESPONSE IN LARGE COMPOSITE STRUCTURES
}

\author{
Oliver Dorn $^{1 *}$, Christian Rolffs ${ }^{1}$, Sven Scheffler ${ }^{1}$, Raimund Rolfes \\ ${ }^{1}$ Leibniz Universität Hannover, Institute of Structural Analysis, Appelstraße 9A, 30167 Hannover, \\ Germany \\ *o.dorn@isd.uni-hannover.de
}

Due to their superior lightweight potential, composites are used in a wide variety of large and slender structures such as gliders, rotor blades of wind turbines or vertical tails of transportation aircrafts. The numerical analysis of these laminated materials typically follows a layer-based approach which describes the stress-strain response of an unidirectional layer within the laminate. Advantages of this method are low experimental characterization costs. The material parameters for an unidirectional layer allow the analysis of arbitrary laminate stackings. In addition, since the position of each layer in the stacking sequence is available, the kinematic behavior of the laminate due to failure of single plies can be predicted accurately in terms of a progressive damage analysis.

On the other hand taking material nonlinearities as failure modes, softening, viscoelasticity or plasticity in a layer-based approach into account often results in an inacceptable computational effort if applied to a large structure. Submodelling or multi-scale-modelling are techniques to tackle this problem $[1,2,3]$.

Even though these techniques or detailed models of critical areas of a structure are a solution for many applications, this is not the case if it comes to the analysis of the change in the aeroelastic response of frp rotor blades due to local damage progression or the investigation of reinforcements on the crash worthiness of an frp glider fuselage, to name two examples.

Most commonly used laminate stackings and sandwich structures can be described by means of a quasi-orthotropic material symmetry. Therefore, in this contribution the finite element framework of a reduced order model for (quasi-) orthotropic materials including nonlinearties as damage and plasticity is presented. The nonlinear material response is derived from virtual tests using material parameters from standard characterization experiments as an input.

The authors believe that this approach is suitable to gain a better understanding of the damage progression, load redistribution phenomena and kinematics in the analysis of large composite structures.

Motivated by the development of a retrofit crash element for gliders, a first attempt to apply the above mentioned method is shown. The individual steps to generate a model are explained. Crash simulations of a glider in comparison to a state of the art finite element modelling approach will be shown.

\section{References}

[1] M. Akterskaia (2019) Global-local progressive failure analysis of composite panels including skin-stringer debonding and intralaminar damage, Ph.D. Thesis, Leibniz University Hannover

[2] S. Hühne (2016) A two-way loose coupling procedure for buckling and damage analysis of composite structures, Ph.D. Thesis, Leibniz University Hannover

[3] G. Ernst (2009) Multiscale Analysis of Textile Composites - Stiffness and Strength -, Ph.D. Thesis, Leibniz University Hannover 